\title{
SERS-based trace detection by size and shape controlled noble metal particles with high benefit-cost ratio
}

Demirtas, Özge, Khan, Ghazanfar Ali , Seyedpour Esmaeilzad, Nasim, Iftikhar, R. M. Faheem, Öztürk, İ. Murat, et al.

Özge Demirtas, Ghazanfar Ali Khan, Nasim Seyedpour Esmaeilzad, R. M. Faheem Iftikhar, İ. Murat Öztürk, A. Kemal Demir, Waqqar Ahmed, Alpan Bek, "SERS-based trace detection by size and shape controlled noble metal particles with high benefit-cost ratio," Proc. SPIE 11797, Plasmonics: Design, Materials, Fabrication, Characterization, and Applications XIX, 1179713 (1 August 2021); doi: 10.1117/12.2594112

SPIE Event: SPIE Nanoscience + Engineering, 2021, San Diego, California, United States 


\title{
SERS-based trace detection by size and shape controlled noble metal particles with high benefit-cost ratio
}

\author{
Özge Demirtaş, ${ }^{\mathrm{a}, \mathrm{b}}$, Ghazanfar Ali Khan ${ }^{\mathrm{c}}$, Nasim Seyedpour Esmaeilzad, ${ }^{\mathrm{a}, \mathrm{b}}$, R. M. Faheem Iftikhar ${ }^{\mathrm{c}}$, \\ İ. Murat Öztürk ${ }^{\text {b,d }}$, A. Kemal Demir ${ }^{\mathrm{e}}$, Waqqar Ahmed ${ }^{\mathrm{c}}$, Alpan Bek*a,b,d \\ ${ }^{a}$ Micro and Nanotechnology Program, Middle East Technical University, 06800 Ankara, Turkey \\ ${ }^{\mathrm{b}}$ Center for Solar Energy Research and Applications (GÜNAM), Middle East Technical University, \\ 06800 Ankara, Turkey \\ ${ }^{\mathrm{c}}$ Materials Laboratory, Department of Physics, COMSATS University, 45500 Islamabad, Pakistan; \\ ${ }^{\mathrm{d}}$ Department of Physics, Middle East Technical University, 06800 Ankara, Turkey \\ e Department of Physics, I. D. Bilkent University, 06800 Ankara, Turkey
}

\section{INTRODUCTON}

SERS has become a highly preferred method with the effect of advances in instrument technology and potential for application to many areas from medicine to art conservation for the detection of trace-level environmental and biological

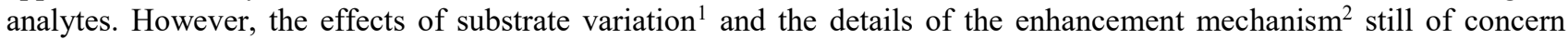
even much progress has been achieved in recent years. Therefore, it is important to control the size and shape of the materials for maximize the enhancement factor and ensure the reproducibility of the substrates by considering SERS uncertainty principle ${ }^{3}$. In this study, we present novel SERS substrate preparation methods that are highly promising in terms of both signal enhancement and reproducibility with high benefit-cost ratio.

\section{METHODOLOGY, RESULTS AND DISCUSSION}

We demonstrate a facile method for trace molecule detection that can be generalized to convert virtually any surface into a SERS substrate. It is within the scope of this study to detect brilliant cresyl blue (BCB) molecules attached to polyol synthesized silver nanoparticles (AgNPs) by demonstrating statistical data for SERS. Figure 1a shows the schematic representation of SERS measurements. Figure 1b shows the SEM image of AgNPs which are spray coated on polished Si wafer. Colloidal AgNPs suspended in ethanol were prepared by polyol synthesis ${ }^{1}$, which is based on the injection of silver nitrate $\left(\mathrm{AgNO}_{3}\right)$ solution, used as a $\mathrm{Ag}$ source, into PVP solution dissolved in ethylene glycol (EG). We have fabricated sparsely decorated $\sim 1-5 \mathrm{~cm}^{2}$ large Si surfaces with AgNPs by spray coating which is a simple and versatile method to convert virtually any macroscopically large surface to a SERS substrate within seconds. The mean value of the AgNP size for all SEM images is calculated as $100 \mathrm{~nm} \pm 5 \mathrm{~nm}$. AgNPs synthesized by the polyol synthesis reveals sharp corners and flat surfaces and end product is covered by $3 \mathrm{~nm}$ thickness of PVP layer as shown in TEM image in Figure 1c. Box charts displaying the statistics of SERS measurements and EFs are shown in Figure 1d, e and f for $10^{-4}$ $\mathrm{M}, 10^{-5} \mathrm{M}$, and $10^{-6} \mathrm{M}$ BCB solution, respectively by two different methods which are (i) AgNP spray coating and BCB solution spin coating, and (ii) spray coating of in-solution mixed AgNPs + BCB. The EF is calculated by the relationship $\mathrm{EF}=\left(\mathrm{I}_{\mathrm{SERS}} / \mathrm{N}_{\mathrm{SERS}}\right) /\left(\mathrm{I}_{\mathrm{NRS}} / \mathrm{N}_{\mathrm{SNRS}}\right)^{3}$ where $\mathrm{N}_{\mathrm{NRS}}$ is the total number of molecules in the measurement volume, $\mathrm{N}_{\mathrm{SERS}}$ is the total number of BCB molecules in the SERS measurement and $\mathrm{I}_{\mathrm{NRS}}$ and $\mathrm{I}_{\mathrm{SERS}}$ values acquired from the area under the peak width of the selected Raman peak of $1419 \mathrm{~cm}^{-1}$. It is evident that the EF values increase with increasing surface coverage values when each column is evaluated individually due to the more frequent presence of inter-particle hotspots. The scattering losses resulting from the surface roughness is eliminated by the atomically flat crystalline surfaces of polyol synthesized AgNPs. Hence, despite the very low surface coverage values, the EF values range from $3.3 \times 10^{5}$ to $2.1 \times 10^{8}$, which are close to the highest EF values obtained through surface averaged calculations in the literature.

For reproducible SERS-based trace molecule detection, we have developed facile synthesis methods of metal nanostructures that ensure highly concentrated hotspots and uniform distribution on the substrate. Firstly, we demonstrated a size-controlled synthesis of multi-spiked gold NPs (MSGNPs) that can be assembled into monolayers over a centimeter length scale simply by drying the suspension on a substrate under ambient conditions ${ }^{6}$. MSGNPs were synthesized by seed-mediated synthesis and our different seed concentrations were added under vigorous stirring, then samples were labeled as from P1 to P4. The average sizes calculated for samples P1, P2, P3, and P4 are 150 $\pm 20,280 \pm$ $30,410 \pm 40$, and $640 \pm 80 \mathrm{~nm}$, respectively, indicating that the size dispersion for all samples is less than $15 \%$. 

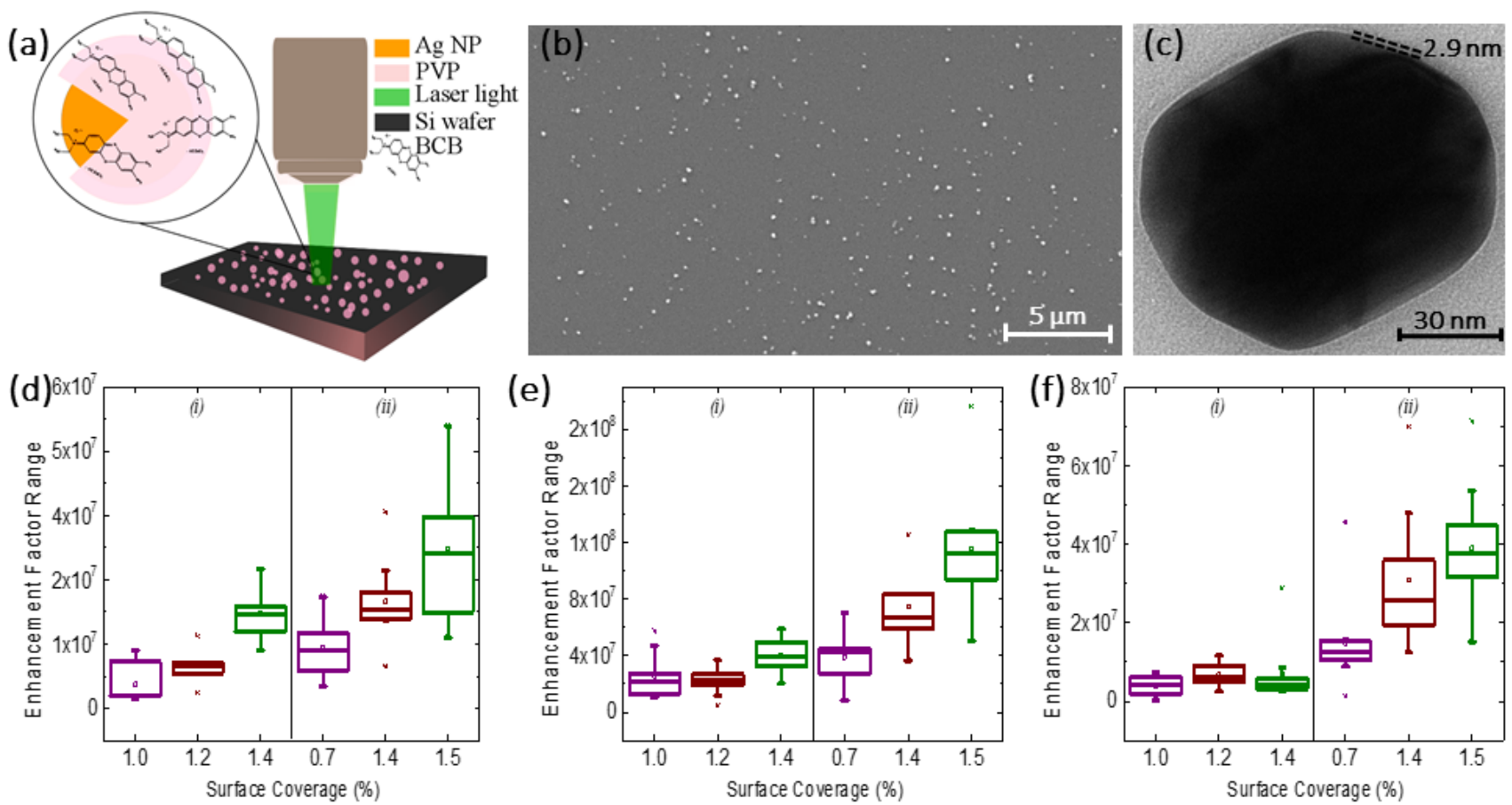

Figure 1: Schematic representation of SERS measurements (a), SEM image of 30 cycles of AgNP spray coating on Si wafers (b), TEM image of a representative polyol synthesized AgNP covered by PVP layer at thickness of $\sim 3 \mathrm{~nm}$ (c), Box charts displaying the statistics of SERS measurements and EFs of (d) $10^{-4} \mathrm{M}$, (e) $10^{-5} \mathrm{M}$, and (f) $10^{-6} \mathrm{M}$ for (i) AgNP spray coating and BCB solution spin coating, and (ii) spray coating of in-solution mixed AgNPs $+\mathrm{BCB}^{5}$.

Figure 2a shows the deposition of the MSGNPs onto silicon wafer; while lower starting particle concentration resulted in coffee ring effect, monolayer assembly of MSGNPs with good homogeneity was obtained by drying the suspension containing a relatively high particle concentration. The fraction of particles making the coffee ring decreased substantially although the thickness and the width of the coffee ring increased. Figure $2 \mathrm{~b}$ shows an SEM image of the monolayer assembly of sample P1. Figure 2c depicts the electric field enhancement simulation for the hexagonal assembly of MSGNPs, corresponding to sample P1. The maximum SERS intensity $\left(\mathrm{E}_{\max } / \mathrm{E}_{0}\right)^{4}$ obtained from simulations for $785 \mathrm{~nm}$ excitation is $8 \times 10^{9}$. The close-packed assembly of MSGNPs further increases the hotspot concentration by generating interparticle hotspots. Furthermore, the calculated experimental enhancement factor for $\mathrm{CV}$ molecule is at the order of $10^{7}$ which is two to three orders of magnitude higher than the reported values for single star-shaped or coresatellite particles ${ }^{7}$.

a

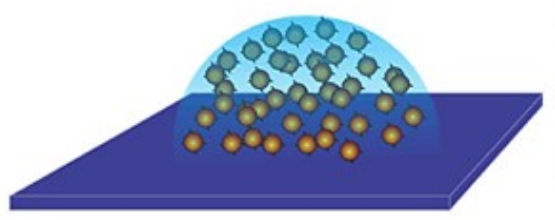

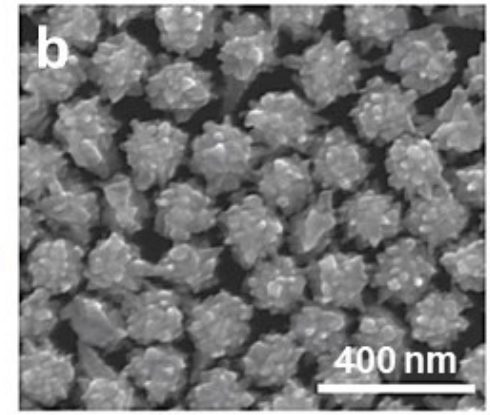

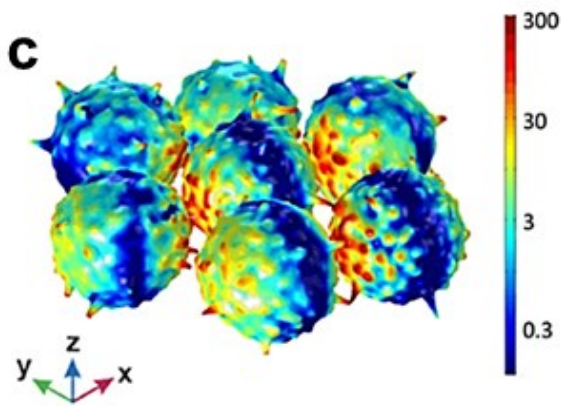

Figure 2: (a) Schematic diagram of the deposition of MSGNP, (b) SEM image of monolayer assembly of sample P1, (c) simulation result of the distribution of the relative electric field of the hexagonal assembly of MSGNPs for sample P $1{ }^{6}$.

Figure 3a shows the results of SERS measurements of various CV molecule concentrations performed to investigate the limit of detection for the sample P1 using $785 \mathrm{~nm}$ excitation source. The SERS platforms fabricated this way reveals that a $\mathrm{CV}$ concentration of as low as $10 \mathrm{fM}$ was measurable. 
Figure $3 b$ shows comparative normalized intensity of $1172 \mathrm{~cm}^{-1}$ peak for different sizes of MSGNPs for different laser excitation wavelengths. It is evident that the enhancement is relatively more pronounced for the smaller NPs for $532 \mathrm{~nm}$ laser excitation source and similarly for larger NPs for longer wavelength excitation source. Hence, maximum field enhancement can be obtained by choosing proper MSGNPs size for available Raman excitation source.
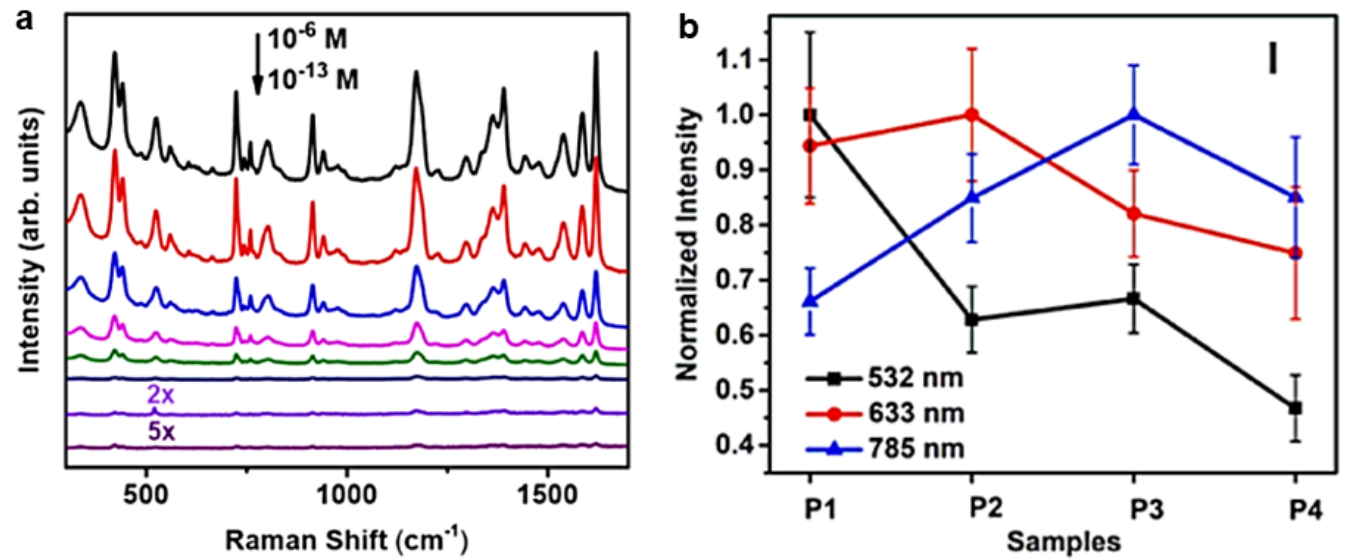

Figure 3: (a) SERS spectra with varying CV concentrations on P1, (b) comparative intensity of $1172 \mathrm{~cm}^{-1}$ peak for different samples with the maximum normalized to 1 , with different excitation sources.

In our second study, the fabrication of Ag nanostructures with worm-like bends and high surface roughness has been demonstrated as highly stable, sensitive, and cost-effective (per- substrate material $=5$ cents) SERS platforms. Surfactant-free and uniformly interconnected branched nanostructure fabrication takes only a few minutes by our method. In-situ fabrication of $\mathrm{Ag}$ nanostructures on the cellulose fibers of the filter paper involves the reduction of the $\mathrm{Ag}$ ions by $\mathrm{NaBH}_{4}$ and subsequent nucleation and growth of nanostructures. Figure 4a depicts a TEM image of the nanostructures transferred to the TEM grid representing inter-connected branched morphology. Figure $4 \mathrm{~b}$ shows Raman spectra of simple filter paper, Ag deposited filter paper substrate (AFPS), and Raman active molecule (CV) coated AgFPS, without background subtractions. The strong fluorescence background of simple filter paper is suppressed, thanks to the bends, surface roughness, and clustering in nanostructures, providing high-density hotspots for electromagnetic field enhancement. Figure 4c depicts the limit of detection studies of SERS substrates, allowing concentrations down to $10^{-8} \mathrm{M}$ to be easily detected. Furthermore, a linear correlation between the intensity and CV concentration has been observed. Finally, the stability of the AgFPS was evaluated by comparing the SERS signal from a freshly prepared substrate with the ones stored for 1 week and 1 month. Figure 4d depicts the corresponding bar chart of average SERS intensity of 1176 $\mathrm{cm}^{-1}$. The measured decrease in the intensity of the peak over one month suggests survival of EF at about $50 \%$ of its original value after 1 year. The supporting filter paper substrate lowers the surface energy of Ag nanostructures by providing stable support, which could be the cause of the extended SERS signal/substrate durability.
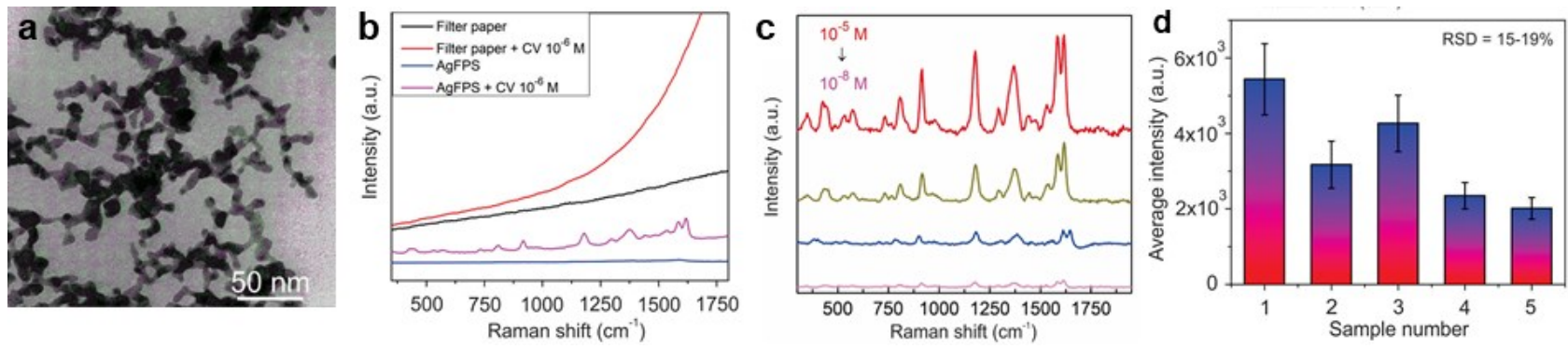

Figure 4: (a) TEM micrograph of interconnected Ag nanostructures transferred to a TEM grid, (b) SERS spectra of filter paper, AgFPS and AgFPS plus $1 \mu \mathrm{M} \mathrm{CV}$, (c) SERS spectra of different concentration of $10 \mu \mathrm{CV}$, (d) the average intensities of $1176 \mathrm{~cm}^{-1}$ peak $^{8}$. 
The seed-mediated method, in which nucleation and growth are decoupled, is often used for the synthesis of Au nanoparticles ${ }^{9,10}$. However, surprisingly, we have seen in our recent study that a higher initial starting concentration of $\mathrm{HAuCl}_{4}$ and ascorbic acid (AA) in the growth solution, nucleation, and subsequent growth can occur in the absence of seed solution. We demonstrate that the shape and size of the gold micro-particles (GMPs) can be tuned simply by changing the concentration of $\mathrm{AA}$ and $\mathrm{CTAB}$ in the growth medium. GMPs were synthesized using a single-step reaction. $\mathrm{AgNO}_{3}$ was added to $\mathrm{HAuCl}_{4}$ and $\mathrm{CTAB}$ solution and then $500 \mu \mathrm{l}, 1 \mathrm{ml}, 2 \mathrm{ml}$, and $5 \mathrm{ml}$ of $0.1 \mathrm{M} \mathrm{AA}$ was added and the samples were labeled as NS1, NS2, NS3, NS4, and NS5, respectively. Finally, to remove excess CTAB, the solution was centrifuged and redispersed in DI water. Change in AA concentration has a great effect on the surface morphology of GMPs as shown in Figure 5. For lower AA concentration, relatively slower growth results in the formation of smoother surface morphology, as is the case for NS1.

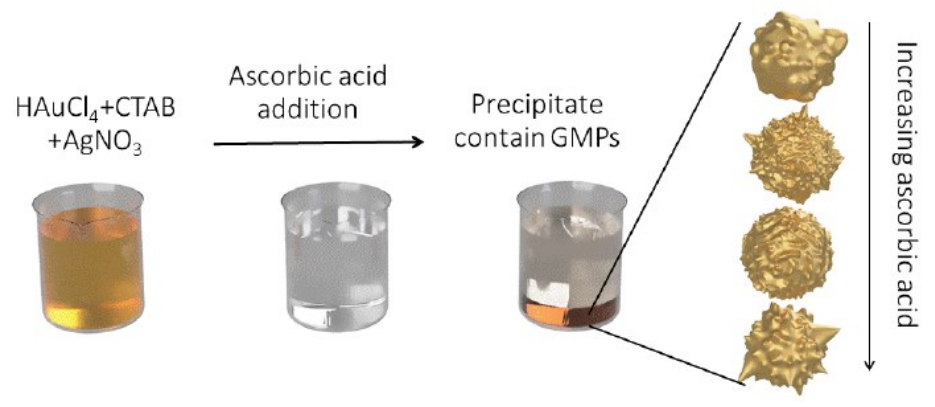

Figure 5: Schematic of shape controlled synthesis of GMPs.

However, the NS2 sample has hundreds of dense short and sharp branches on the particle's surface resulting in hedgehog-like morphology as shown in the SEM image in Figure 6a. The increase in the AA concentration results in an accelerated autocatalytic reduction, specifically at higher energy defect sites which are more suitable for the deposition of incoming $\mathrm{Au}$ monomers. Faster growth kinetics leads to the formation of branches originating from these defect sites (samples NS2 and NS3). However, further increase in AA may result in the growth kinetics being so high that the monomers react/deposit comparatively uniformly everywhere on the surface of growing particles, resulting in relatively uniform surface morphology (sample NS4 and NS5). Finite-element simulations indicate that the most intense electromagnetic enhancement is seen to be at the tips of the branches. The highest number of hotspots is observed for NS2 due to the presence of the highest number of branches as shown in Figure $6 \mathrm{c}$ and the maximum SERS enhancement is obtained as in excess of $10^{9}$. SERS spectra of different GMPs using CV molecule as the analyte is shown in Figure $6 \mathrm{~d}$. The highest signal is collected from NS2, the lowest signal is from NS1. These results are consistent with the simulation results, which predicted the concentration of hotspots for NS2 and the lowest for sample NS1 (Figure 6b). The small surface protrusions have shown better enhancement for shorter laser wavelengths, while longer branches exhibit more pronounced enhancement for longer excitation wavelengths. Figure 6e depicts the SERS spectra of CV molecule obtained from NS2 under different excitation laser wavelengths. Although the enhancement seems to be different for different excitation wavelengths, due to different concentrations of hotspots for a particular excitation, SERS peak intensities in all cases is quite good. Therefore, GMPs have great potential to become a universal SERS substrate.
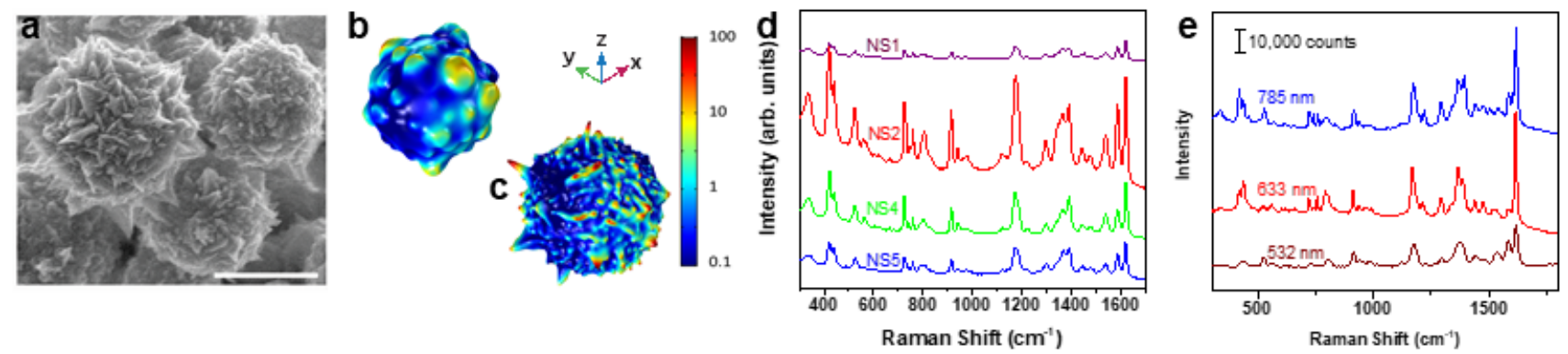

Figure 6: SEM images of sample NS2 (a), simulation results of the relative electric field distribution of sample NS1 (b), NS2 (c), SERS spectra of $1 \mu \mathrm{M} \mathrm{CV}$ deposited on different GMPs (d), SERS spectra of $1 \mu \mathrm{M} \mathrm{CV}$ obtained at different laser wavelengths (e). In addition to the exclusive chemical synthesis techniques aforementioned, another effective approach is employing metal film-over-nanosphere (FON) for SERS-based trace detection. FON based SERS substrate design includes studying 
effects of bimetallic coating on microsphere arrays as a function of coating thickness ${ }^{11}$, constant metal film thickness on nanospheres as a function of dielectric sphere diameter ${ }^{12}$. Our study shows a novel approach that includes ultra-thin $\mathrm{Ag}$ film deposition at low tilt-angles on a shape-modified NSL mask. The solution of colloidal PS NSs was spin-coated on the Si samples. Figure 7a displays the top-view SEM image of the self-organized $350 \mathrm{~nm}$ size PS-NSs in which hcp configuration is clearly visible. To improve the adhesion of PS NSs to the Si surface, self-organized PS NSs annealed at $110^{\circ} \mathrm{C}$ for 20 minutes on a hot plate. Figure $7 \mathrm{~b}$ shows a side-view image of NSs after thermal treatment. Next, two different thicknesses of $10 \mathrm{~nm}$ and $20 \mathrm{~nm} \mathrm{Ag}$ deposition at incidence angles of $\theta=0^{\circ}, 5^{\circ}, 10^{\circ}, 15^{\circ}$ is performed on NSL mask, and fabrication of AgFON array is finalized as shown in Figure 7c. Lastly, CV molecules are spin-coated on an AgFON array from an aqueous solution at $10^{-4} \mathrm{~mol} / \mathrm{L}$ concentration for characterization of Raman measurements with $532 \mathrm{~nm}$. COMSOL Multiphysics was employed to carry out FEM based Maxwell simulations. Figure 7d shows the maximum Raman EF (based on approximation of $\mathrm{EF}=\mathrm{E}_{\mathrm{loc}}{ }^{4} / \mathrm{E}_{0}{ }^{4}$ approximation) as a function of polarization angle for different deposition angles. While the Raman $\mathrm{EF}$ is maximum for $\theta=5^{\circ}$ for all incidence polarizations, a significant decrease in $\mathrm{EF}$ is observed for $\theta>5^{\circ}$ which can be explained by the variation in the shape and coverage of $\mathrm{Ag}$ film.
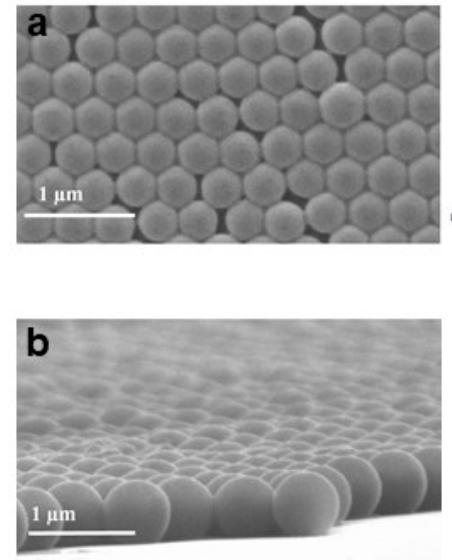
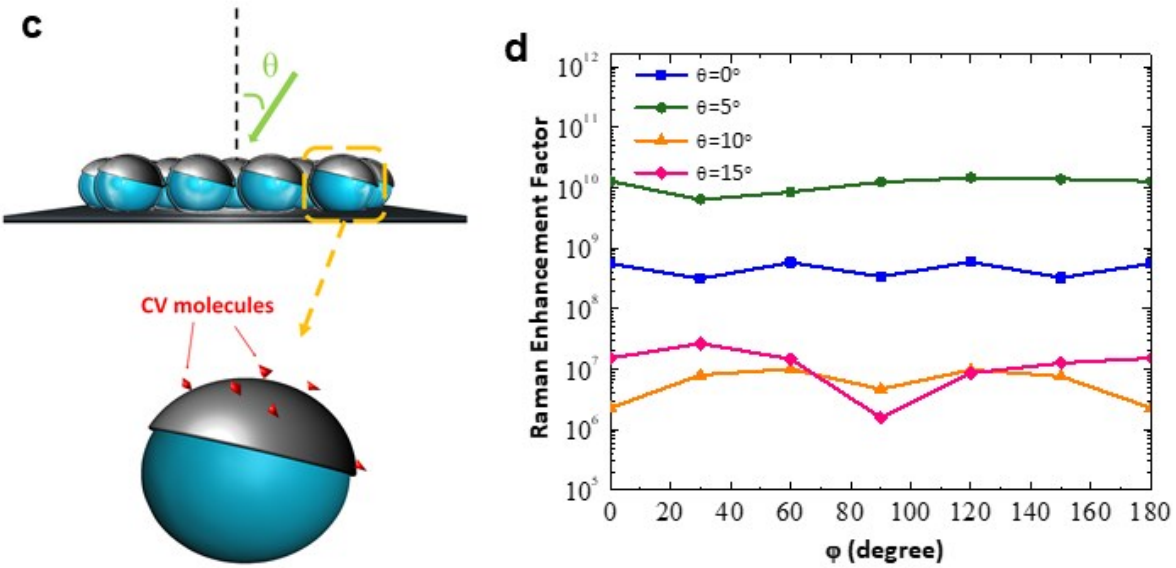

Figure 7: (a) SEM image of as-deposited hcp PS NSs with $350 \mathrm{~nm}$ size in top view, (b) SEM image of 20 min $110^{\circ} \mathrm{C}$ annealed PS NSs in side-view, (c) Oblique angle deposition of an Ag film over PS NSs and nanosphere deposited with CV molecule, (d) Raman EF calculated using FEM simulations of AgFONs deposited at various $\theta$ as a function of incident polarization angle.

Figure 8a shows the experimentally determined EFs where statistics of SERS measurements cover spectra acquired from 10 different random spots on each substrate prepared at different $\theta$ and $\mathrm{Ag}$ coating thicknesses before and after annealing of PS NSs. At $20 \mathrm{~nm}$ thickness of Ag, heat treatment and tilt angle have no statistically significant effect in the EF.
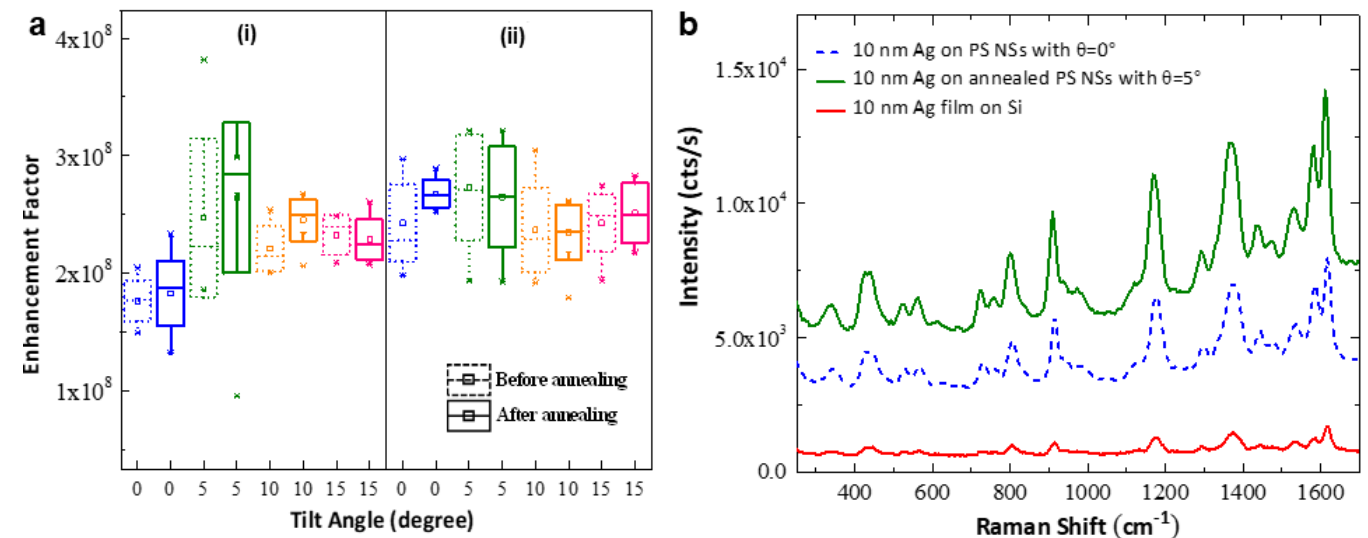

Figure 8: (a) Box charts displaying the population statistics of SERS EFs of (i) $10 \mathrm{~nm}$ and (ii) $20 \mathrm{~nm}$ Ag coated PS NSs before (dotted lines) and after (solid lines) annealing with $\theta$ values of $0^{\circ}, 5^{\circ}, 10^{\circ}$ and $15^{\circ}$, (b) SERS spectra of $10^{-4} \mathrm{M} \mathrm{CV}$ spin-coated on (b) $10 \mathrm{~nm}$ $\mathrm{Ag}$ on non-annealed PS NSs (dotted blue line), annealed PS NSs with $\theta=5^{\circ}$ (solid green line), and $10 \mathrm{~nm} \mathrm{Ag} \mathrm{coating} \mathrm{on} \mathrm{Si} \mathrm{(solid} \mathrm{red}$ line). 
In fact, statistics show that maximum EF is obtained from $10 \mathrm{~nm} \mathrm{Ag}$ coating with $\theta=5 \mathrm{o}$ on annealed PS NSs. It is evident that with heat treatment and oblique angle deposition high EF SERS substrates can be obtained with the advantage of material cost reduction such that at least half the amount of Ag material can be saved. Figure $8 \mathrm{~b}$ shows the averaged SERS spectra. Shape adjustment of the NSs by thermal treatment together with Ag deposition at $\theta=50$ enhances the Raman signal as compared to the $\theta=5 \mathrm{o}$ without annealing process. In addition, at the same $\mathrm{Ag}$ coating thickness of 10 $\mathrm{nm}, 103$ times Raman intensity enhancement is acquired on NSs with respect to flat Ag film on Si.

\section{CONCLUSIONS}

We have demonstrated SERS activity of AgNPs by spray coating method which is especially chosen for its simplicity and versatility to convert virtually any macroscopically large surface to a SERS substrate within seconds. Unlike available SERS substrates with $\sim 100 \%$ surface coverage with noble metals, our AgNPs need to cover as low as only $\sim 1 \%$ of the surface in order to produce EFs as high as $10^{8}$. We have also demonstrated that highly uniform and efficient SERS substrates can be fabricated by making monolayer close-packed assemblies of MSGNPs of various sizes simply by drying a high concentration of MSGNP suspension under ambient conditions. In addition, we reported stable and efficient SERS substrates with the advantage in-situ growth of surfactant-free Ag nanostructures. This can be attributed to the reduction of the surface energy of Ag NPs through supporting substrate. We demonstrated a novel method regarding the synthesis of shape and size controlled GMPs enabling it to be effective for SERS done by using different excitation sources. Lastly, we presented a cost-effective fabrication method of Ag FON SERS substrate by tuning the shape and deposition angle. With this method, we show that ultra-thin Ag films deposited at $5^{\circ}$ tilt can replace SERS substrates fabricated by deposition of $\mathrm{Ag}$ films with double thickness in normal deposition angle.

\section{ACKNOWLEDGMENTS}

Authors thank Higher Education Commission (HEC) Pakistan and the Council of Higher Education (YÖK) Turkey for Pak-Turk Research Mobility Grant 2018 under grant nr. 1550. Ö.D. and A.B. thanks The Scientific and Technological Research Council of Turkey (TUBITAK) for partial financial support under 2211-C program and grant nr. 119N413 and 119F101, respectively. Ö.D. thanks the Council of Higher Education (YÖK) for support under 100/2000 program.

\section{REFERENCES}

[1] Rycenga, M., Cobley, C. M., Zeng, J., Li, W., Moran, C. H., Zhang, Q., Qin, D. and Xia, Y., "Controlling the Synthesis and Assembly of Silver Nanostructures for Plasmonic Applications," Chem. Rev. 111(6), 3669-3712 (2011).

[2] Le Ru, E. C., Blackie, E. J., Meyer, M., Etchegoin, P. G. and Blackie, E. J., "Surface Enhanced Raman Scattering Enhancement Factors: A Comprehensive Study,” J. Phys. Chem. C 111(37), 13794-13803 (2007).

[3] Snežzana Miljanić, Leo Frkanec, Tomislav Biljan, 3 Zlatko Meić Mladen Žini ć., "Recent Advances in linear and nonlinear Raman spectroscopy I,” J. Raman Spectrosc. 38(April), 1538-1553 (2007).

[4] Coskun, S., Aksoy, B. and Unalan, H. E., "Polyol Synthesis of Silver Nanowires: An Extensive Parametric Study," Cryst. Growth Des. 11(11), 4963-4969 (2011).

[5] Demirtaş, Ö., Doğanay, D., Öztürk, İ. M., Ünalan, H. E. and Bek, A., "Facile preparation of nanoparticle based SERS substrates for trace molecule detection," Phys. Chem. Chem. Phys. 22(37), 21139-21146 (2020).

[6] Ahmed, W., Demirtaş, Ö., Öztürk, İ. M. and Bek, A., "Monolayer Assembly of MultiSpiked Gold Nanoparticles for Surface-Enhanced Raman Spectroscopy-Based Trace Detection of Dyes and Explosives,” ACS Appl. Nano Mater. 3(7), 6766-6773 (2020).

[7] Tran, V., Thiel, C., Svejda, J. T., Jalali, M., Walkenfort, B., Erni, D. and Schlücker, S., "Probing the SERS brightness of individual $\mathrm{Au}$ nanoparticles, hollow $\mathrm{Au} / \mathrm{Ag}$ nanoshells, $\mathrm{Au}$ nanostars and $\mathrm{Au}$ core/Au satellite particles: single-particle experiments and computer simulations," Nanoscale 10(46), 21721-21731 (2018).

[8] Khan, G. A., Demirtaş, Ö., Demir, A. K., Aytekin, Ö., Bek, A., Bhatti, A. S. and Ahmed, W., "Fabrication of flexible, cost-effective, and scalable silver substrates for efficient surface enhanced Raman spectroscopy based trace detection," Colloids Surfaces A Physicochem. Eng. Asp. 619(March), 126542 (2021).

[9] Jana, N. R., Gearheart, L. and Murphy, C. J., "Seed-Mediated Growth Approach for Shape-Controlled Synthesis of Spheroidal and Rod-like Gold Nanoparticles Using a Surfactant Template," Adv. Mater. 13(18), 1389-1393 
(2001).

[10] Liu, M. and Guyot-Sionnest, P., "Mechanism of Silver(I)-Assisted Growth of Gold Nanorods and Bipyramids," J. Phys. Chem. B 109(47), 22192-22200 (2005).

[11] Wang, J., Zhou, F., Duan, G., Li, Y., Liu, G., Su, F. and Cai, W., "A controlled Ag-Au bimetallic nanoshelled microsphere array and its improved surface-enhanced Raman scattering effect," RSC Adv. 4(17), 8758 (2014).

[12] Greeneltch, N. G., Blaber, M. G., Schatz, G. C. and Van Duyne, R. P., "Plasmon-Sampled Surface-Enhanced Raman Excitation Spectroscopy on Silver Immobilized Nanorod Assemblies and Optimization for Near Infrared $(\lambda$ ex $=1064$ nm) Studies," J. Phys. Chem. C 117(6), 2554-2558 (2013). 